\title{
Original
}

\section{The association between daily calcium intake and sarcopenia in older, non-obese Korean adults: the fourth Korea national health and nutrition examination survey (KNHANES N) 2009}

\author{
Mi Hae Seo ${ }^{1)^{*}}$, Mee Kyoung Kim ${ }^{2)^{*}}$, Se Eun Park ${ }^{1)}$, Eun Jung Rhee ${ }^{1)}$, Cheol Young Park ${ }^{1)}$, \\ Won-Young Lee ${ }^{1)}$, Ki Hyun Baek ${ }^{2)}$, Ki-Ho Song ${ }^{2)}$, Moo Il Kang ${ }^{2)}$ and Ki Won Oh ${ }^{1)}$ \\ 1) Division of Endocrinology and Metabolism Department of Internal Medicine, Kangbuk Samsung Hospital, Sungkyunkwan \\ University School of Medicine, Seoul, Korea \\ 2) Division of Endocrinology and Metabolism, Department of Internal Medicine, The Catholic University of Korea, Seoul, Korea
}

\begin{abstract}
Recent data suggest that variations in calcium intake may influence body weight and composition; however, the relationship between daily calcium intake and muscle mass has not been well established. The objective of this study was to assess the relationship between daily calcium intake and sarcopenia. We analyzed data for older adults (over 60 years) from the fourth Korea National Health and Nutrition Examination Survey (KNHANES) conducted in 2009. A total of 1339 Non-Obese (BMI between 18.5 and $25 \mathrm{~kg} / \mathrm{m}^{2}$ ), older adults (592 men and 707 women) were enrolled. Dietary variables were assessed using a nutrition survey that used a 24-hour recall method. Daily calcium intake based on the consumption of each food item was calculated. Sarcopenia was defined as an appendicular skeletal muscle mass divided by body weight less than 2 SD below the sex-specific mean for young adults. We found that daily calcium intake was negatively correlated with total body fat percentage and positively correlated with appendicular skeletal mass $(p<0.001)$. Participants with sarcopenia appear to have significantly lower daily calcium intakes than participants without sarcopenia $(p<0.001)$. The unadjusted prevalence of sarcopenia according to daily calcium intake tertiles were $6.3 \%, 4.3 \%$, and $2.7 \%$ in tertiles 1,2 , and 3, respectively. After adjustment for age, sex, BMI, total energy intake, and lifestyle factors, compared with those in the lowest tertile of daily calcium intake, participants in the highest tertile had an odds ratio for sarcopenia of $0.295(95 \%$ confidence interval, $0.087-0.768 ; p$ for trend $=0.014$ ). We found that daily calcium intake, corrected for total energy intake and serum $25(\mathrm{OH}) \mathrm{D}$ status, was significantly lower in subjects with sarcopenia than in those without. Our results suggest a strong inverse association between daily calcium intake and sarcopenia in non-obese, older Korean adults.
\end{abstract}

Key words: Daily calcium intake, Sarcopenia, Non-Obese older adult

A NUMBER of epidemiological studies have shown a strong inverse correlation between calcium intake and adiposity. Individuals with the lowest calcium intake tend to have the highest body weight [1-3]. Data from the National Nutrition Survey 1995 indicate that Australian men and women with higher calcium intakes have lower BMIs and waist circumferences, after controlling for various confounders. More recent clinical studies have documented that a diet high in

Submitted Nov. 1, 2012; Accepted Jan. 6, 2013 as EJ12-0395

Released online in J-STAGE as advance publication Jan. 26, 2013

Correspondence to: Ki Won Oh, M.D., Ph.D., Department of Internal Medicine, Kangbuk Samsung Hospital, Sungkyunkwan University School of Medicine, 108 Pyung-dong, Chongro-Ku, Seoul 110-746, Korea. E-mail: okwendo@naver.com

* These authors have contributed equally to the work. dairy calcium accelerates fat loss, particularly from the abdominal region, and that increasing calcium intake is a potential means of weight management [4-6]. In particular, high calcium intakes were found to decrease the rate of lipogenesis and enhance lipolysis in adipocytes $[7,8]$. In contrast, there have been no studies of the relationship between muscle mass and daily calcium intake.

Numerous studies have shown age-related body composition changes in both men and women, notably that fat mass increases and muscle mass decreases with age $[9,10]$. Sarcopenia is an evolving concept and the current definition includes both a loss of muscle strength and loss of muscle mass [11]. The etiology of sarcopenia is multifactorial. Factors that have been implicated in sarcopenia include a decreased 
physical activity level, declining androgen concentrations, chronic disease, inflammation, insulin resistance, and nutritional deficiencies (dietary protein and serum 25(OH)D) [12]. Importantly, cachexia and obesity may also contribute to sarcopenia. Hence, excluding these subpopulations (underweight, overweight, obese) might be of clinical importance for assessing the risk factors of sarcopenia.

The aim of our study was to investigate whether dietary calcium intake is associated with sarcopenia in non-obese, older Korean adults.

\section{Subjects and Methods}

\section{Study population}

This cross-sectional study was based on data acquired by the Fourth Korea National Health and Nutrition Examination Survey (KNHANES IV) conducted in 2009. The KNHANES surveys have been conducted periodically since 1998 to assess the health and nutritional status of the non-institutionalized civilian population of Korea [13]. KNHANES IV was a cross-sectional and nationally-representative survey conducted by the Division of Chronic Disease Surveillance, Korea Centers for Disease Control and Prevention, from 2007-2009. There were 2363 subjects aged $60 \mathrm{yr}$ or older among those who participated in the survey between January and December 2009. To confine the study sample to non-obese adults, the data used in our study were limited to participants with body mass indexes (BMIs) between 18.5 and $25 \mathrm{~kg} / \mathrm{m}^{2}$ (BMI over $25 \mathrm{~kg} / \mathrm{m}^{2}, \mathrm{n}=751$ ).

We excluded individuals without data for oral daily calcium intake $(n=154)$ and those with a history of chronic kidney disease $(n=10)$. After excluding the non-eligible subjects, the total number of participants for this analysis was 1339 (632 men and 707 women). All the participants in this survey signed an informed consent form.

\section{Clinical and laboratory measurements}

Dietary variables were assessed using a 24-hour recall method in the nutrition survey. Daily intakes of total energy, fat, fiber, and calcium were calculated based on the food items consumed.

Blood samples were collected from each participant during the survey after a 12-hour overnight fast, and were processed, immediately refrigerated, and transported in cold storage to the Central Testing Institution.
Serum levels of glucose, total cholesterol (TC), highdensity lipoprotein (HDL) cholesterol, triglycerides (TG), creatinine, aspartate aminotransferase (AST), and alanine aminotransferase (ALT) were measured enzymatically using a Hitachi automatic analyzer 7600 (Tokyo, Japan). Serum 25(OH)D concentrations were measured by RIA (DiaSorin Inc., Stillwater, MN, USA) using a counter (1470 Wizard; PerkinElmer, Turku, Finland).

A parathyroid hormone (PTH) assay was performed in the same laboratory using a chemiluminescence assay (DiaSorin) for the measurement of intact PTH. Insulin concentrations were measured by immunoradiometry (INS-IRMA; Bio-Source, Nivelles, Belgium). The homeostasis model assessment estimate of insulin resistance (HOMA-IR) was calculated using the following formula: fasting plasma glucose (millimoles per liter)/fasting insulin (milli-IU per liter)/22.5 [14].

\section{Body composition}

Body mass index (BMI) was calculated as weight/ height ${ }^{2}\left(\mathrm{~kg} / \mathrm{m}^{2}\right)$ and waist circumference was measured at the midpoint between the lower border of the rib cage and the iliac crest. A whole body DXA scan was performed for each subject to measure total and regional lean mass $(\mathrm{kg})$, total body fat $(\mathrm{kg})$, and total body fat percentage (\%) using fanbeam technology (Hologic; Bedford, MA, USA). Appendicular skeletal muscle mass (ASM (kg)) was defined as the sum of lean soft tissue masses for the arms and legs, following the method of Heymsfield et al. [15]. We used ASM as a percentage of body weight (Wt), as modified in studies by Janssen et al. [16] and Lim et al. [17].

\section{Definition of sarcopenia}

Sarcopenia was defined as an appendicular skeletal muscle mass divided by body weight (ASM/Wt) less than 2 SD below the sex-specific mean of a sample of 2392 healthy adults aged 20-40 yr (1054 men, 1338 women) $[17,18]$. For men, the cutoff value for sarcopenia was $29.5 \%$ (ASM/Wt); for women, the corresponding cutoff value was $23.2 \%$ (ASM/Wt).

\section{Statistical analysis}

Continuous variables were reported as mean $\pm \mathrm{SD}$ and compared by independent $t$ tests. Categorical variables were expressed as percentages and compared using a $\chi^{2}$ test. Spearman correlation coefficients between daily calcium intake and various parameters of body compo- 
sition were calculated. The participants' characteristics were compared according to sarcopenia status using independent-sample Student's $t$ tests.

Multiple logistic regression analysis was conducted to evaluate associations between daily calcium intake and sarcopenia. The participants were classified into daily calcium intake tertiles (T1, T2, and T3). The odds ratios (OR) and 95\% confidence intervals (CI) were calculated. We used multiple logistic regression analysis to determine the OR of sarcopenia in subjects stratified by tertiles of daily calcium intake. Adjustments were made for the following variables: age, sex, BMI, total energy intake, serum 25(OH)D level, PTH, HOMA-IR, regular exercise, occupation, region, smoking, alcohol drinking, and vitamin and mineral supplement use. For testing linear risk trends, we used the tertile of daily calcium intake as a continuous variable in the regression model. A value of $p<0.05$ was accepted as significant. All data were analyzed using IBM SPSS Statistics 20.0 (IBM, Armonk, NY, USA).

\section{Results}

\section{General characteristics of the study participants}

The general characteristics of the study participants according to daily calcium intake tertiles are shown in Table 1. The cross-sectional analyses included data on 1339 participants (592 men and 707 women). The mean age of the study participants was $70.1 \mathrm{yrs}$. When we compared participant characteristics across daily calcium intake, sex, and age, we found that total body fat mass and total body fat percentage decreased with increasing daily calcium intake. There were no significant differences in lipid and glucose profiles according to daily calcium intake tertile. BMI, waist circumference, ASM, ASM/Wt (\%), regular exercise, total energy intake, and protein ( $\%$ of energy) were significantly different and increased with increasing daily calcium intake tertiles.

Table 2 presents metabolic and dietary characteristics according to sarcopenia status. The mean daily calcium intake was significantly lower in those with sarcopenia than in those without sarcopenia (316.37 \pm 252.39 vs. $414.65 \pm 316.10 \mathrm{mg} /$ day, $p=0.005$; Table 2). The prevalence of known diabetes mellitus and hypertension was significantly higher in subjects with sarcopenia. Subjects with sarcopenia were older, more obese, and were less likely to exercise regularly than those without sarcopenia. Subjects with sarcopenia had greater total body fat mass and lower ASM. There were no significant differences in lipid profiles, serum fasting glucose levels, and HOMA-IR values between the sarcopenia and non-sarcopenia groups (Table 2).

Spearman correlation analysis showed that daily calcium intake correlated negatively with total body fat percentage (rho $=-0.140, p<0.001$ ). Daily calcium intake correlated positively with truncal lean body mass ( rho $=$ $0.280), \mathrm{ASM}(\mathrm{rho}=0.276), \mathrm{ASM} / \mathrm{Wt}(\mathrm{rho}=0.182)$, and total body lean body mass $($ rho $=0.281)($ all $p<0.001)$. In addition, according to the Spearman correlation analysis, bone mineral density (BMD) of the femur neck correlated positively with ASM and ASM/Wt (ASM, rho $=0.693, p<0.001$; ASM/Wt rho $=0.463, p<0.001)$.

\section{Prevalence of sarcopenia according to daily calcium intake tertile}

As seen in Fig. 1, the proportion of subjects with sarcopenia significantly decreased as daily calcium intake increased from the first to the third tertile $(p$ for trend $=$ $0.009)$. The point estimates for prevalence of sarcopenia were $6.3,4.3$, and $2.7 \%$ in $\mathrm{T} 1, \mathrm{~T} 2$, and $\mathrm{T} 3$, respectively.

\section{Risk of sarcopenia according to daily calcium intake tertile}

When the odd ratios for sarcopenia according to daily calcium intake tertile were analyzed with logistic regression analyses, the risk for sarcopenia decreased as daily calcium intake increased from the first to the third tertile, after correction for confounding variables. After adjustment for age, sex, and BMI, the OR for having sarcopenia was $0.380(95 \% \mathrm{CI}=0.183-0.790)$ in subjects in T3 for daily calcium intake $(\geq 444.5 \mathrm{mg} /$ day) compared with those in $\mathrm{T} 1(\leq 241.8 \mathrm{mg} /$ day) $(p$ for trend $=0.008$; Model 2, Table 3). Controlling further for alcohol consumption, smoking, regular exercise, occupation, serum 25(OH)D levels, HOMA-IR, and lifestyle factors did not attenuate the association between daily calcium intake and sarcopenia $(\mathrm{OR}=$ $0.391 ; 95 \% \mathrm{CI}=0.163-0.940 ; p$ for trend $=0.033$ ). The association remained significant after further adjustment for serum parathyroid hormone level and total fat mass; however, T2 did not show a significantly decreased risk for sarcopenia in all adjustment models. Thus, there appears to be a threshold effect in that sarcopenia was only decreased in the highest tertile ( $\geq 444.5 \mathrm{mg} /$ day). 
Table 1 Clinical characteristics of participants according to daily calcium intake

\begin{tabular}{|c|c|c|c|c|}
\hline \multirow[b]{2}{*}{ Variable } & \multicolumn{3}{|c|}{ Daily oral calcium intake tertile } & \multirow[b]{2}{*}{$p$ for trend } \\
\hline & $\begin{array}{c}\mathrm{T} 1(\leq 241.5 \mathrm{mg} / \mathrm{dL}) \\
\mathrm{N}=447\end{array}$ & $\begin{array}{c}\mathrm{T} 2(241.6-444.4 \mathrm{mg} / \mathrm{dL}) \\
\mathrm{N}=445\end{array}$ & $\begin{array}{c}\mathrm{T} 3(\geq 444.5 \mathrm{mg} / \mathrm{dL}) \\
\mathrm{N}=447\end{array}$ & \\
\hline Sarcopenia & $28(6.3 \%)$ & $19(4.3 \%)$ & $12(2.7 \%)$ & 0.009 \\
\hline Age (yr) & $72.0 \pm 6.8^{\mathrm{a}, \mathrm{b}}$ & $69.6 \pm 6.6^{\mathrm{a}, \mathrm{c}}$ & $68.6 \pm 6.3^{\mathrm{b}, \mathrm{c}}$ & $<0.001$ \\
\hline Sex (Male) & $155(34.7 \%)$ & $215(48.3 \%)$ & $262(58.6 \%)$ & $<0.001$ \\
\hline BMI $\left(\mathrm{kg} / \mathrm{m}^{2}\right)$ & $21.82 \pm 2.08^{\mathrm{a}, \mathrm{b}}$ & $22.17 \pm 2.09^{\mathrm{a}, \mathrm{c}}$ & $22.30 \pm 1.89^{\mathrm{b}, \mathrm{c}}$ & 0.001 \\
\hline Waist circumference $(\mathrm{cm})$ & $78.67 \pm 7.55^{\mathrm{b}}$ & $79.68 \pm 7.38$ & $80.32 \pm 7.01^{\mathrm{b}}$ & 0.003 \\
\hline Total body fat mass (kg) & $14.3 \pm 4.2$ & $14.4 \pm 4.2$ & $14.0 \pm 4.0$ & 0.492 \\
\hline Total body fat percentage $(\%)$ & $27 . \pm 7.05^{\mathrm{a}, \mathrm{b}}$ & $26.18 \pm 7.35^{\mathrm{a}, \mathrm{c}}$ & $25.02 \pm 7.14^{\mathrm{b}, \mathrm{c}}$ & $<0.001$ \\
\hline $\operatorname{ASM}(k g)$ & $15.5 \pm 3.4^{\mathrm{a}, \mathrm{b}}$ & $17.0 \pm 3.9^{\mathrm{a}, \mathrm{c}}$ & $17.9 \pm 4.0^{\mathrm{b}, \mathrm{c}}$ & $<0.001$ \\
\hline ASM/Wt (\%) & $29.41 \pm 3.89^{\mathrm{a}, \mathrm{b}}$ & $30.40 \pm 4.24^{\mathrm{a}, \mathrm{c}}$ & $31.18 \pm 4.11^{\mathrm{b}, \mathrm{c}}$ & $<0.001$ \\
\hline Total cholesterol (mg/dL) & $193.47 \pm 37.98^{b}$ & $190.03 \pm 35.67$ & $186.86 \pm 36.39^{b}$ & 0.035 \\
\hline HDL-cholesterol (mg/dL) & $49.63 \pm 12.39$ & $50.88 \pm 12.45$ & $50.22 \pm 11.55$ & 0.332 \\
\hline Triglycerides (mg/dL) & $146.52 \pm 108.49$ & $135.20 \pm 79.43$ & $135.50 \pm 86.47$ & 0.133 \\
\hline Insulin $(\mu \mathrm{IU} / \mathrm{mL})$ & $9.56 \pm 11.7$ & $8.64 \pm 4.23$ & $8.54 \pm 3.34$ & 0.096 \\
\hline HOMA-IR & $2.62 \pm 5.85$ & $2.18 \pm 1.55$ & $2.29 \pm 1.24$ & 0.180 \\
\hline $\operatorname{ALP}(\mathrm{IU} / \mathrm{L})$ & $257.74 \pm 73.76$ & $250.97 \pm 73.17$ & $254.21 \pm 87.52$ & 0.463 \\
\hline AST (IU/L) & $23.83 \pm 12.40$ & $24.19 \pm 14.55$ & $26.44 \pm 25.57$ & 0.088 \\
\hline $\operatorname{ALT}(\mathrm{IU} / \mathrm{L})$ & $19.03 \pm 12.33^{\mathrm{b}}$ & $19.45 \pm 12.02$ & $21.52 \pm 16.54^{b}$ & 0.020 \\
\hline $25(\mathrm{OH}) \mathrm{D}(\mathrm{ng} / \mathrm{mL})$ & $20.05 \pm 7.59$ & $20.23 \pm 7.21$ & $20.70 \pm 7.28$ & 0.420 \\
\hline PTH (ng/liter) & $72.43 \pm 36.64^{\mathrm{b}}$ & $70.46 \pm 28.52$ & $65.37 \pm 28.29^{b}$ & 0.004 \\
\hline Previous hypertension (\%) & $189(42.4 \%)$ & $165(37.1 \%)$ & $189(42.4 \%)$ & 0.176 \\
\hline Previous diabetes mellitus (\%) & $63(14.1 \%)$ & $45(10.1 \%)$ & $84(18.8 \%)$ & 0.045 \\
\hline Previous stroke $(\%)$ & $23(5.2 \%)$ & $22(4.9 \%)$ & $19(4.3 \%)$ & 0.531 \\
\hline Regular exercise ${ }^{\mathrm{A}}(\%)$ & $32(7.3 \%)$ & $43(9.7 \%)$ & $66(14.9 \%)$ & $<0.001$ \\
\hline Vitamin supplement use (\%) & $71(15.9 \%)$ & $98(22.1 \%)$ & $115(25.8 \%)$ & $<0.001$ \\
\hline Region, urban area (\%) & $184(41.2 \%)$ & $241(54.2 \%)$ & $260(58.2 \%)$ & $<0.001$ \\
\hline \multicolumn{5}{|l|}{ Occupation ${ }^{\mathrm{B}}$} \\
\hline Clerical work (\%) & $19(4.3 \%)$ & $25(5.6 \%)$ & $28(6.3 \%)$ & 0.037 \\
\hline Manual work (\%) & $134(30.5 \%)$ & $192(43.3 \%)$ & $154(34.8 \%)$ & 0.037 \\
\hline Unemployed (\%) & $287(65.2 \%)$ & $226(51.0 \%)$ & $260(58.8 \%)$ & 0.037 \\
\hline \multicolumn{5}{|l|}{ Dietary intake $^{\mathrm{C}}$} \\
\hline Total energy (kcal/d) & $1224.88 \pm 434.79^{\mathrm{a}, \mathrm{b}}$ & $1688.29 \pm 534.61^{\mathrm{a}, \mathrm{c}}$ & $2008.57 \pm 764.13^{b, c}$ & $<0.001$ \\
\hline Protein (\% of energy) & $10.9^{\mathrm{a}, \mathrm{b}}$ & $12.8^{\mathrm{a}, \mathrm{c}}$ & $15.0^{\mathrm{b}, \mathrm{c}}$ & $<0.001$ \\
\hline Fat (\% of energy) & $8.7^{\mathrm{a}, \mathrm{b}}$ & $11.4^{\mathrm{a}, \mathrm{c}}$ & $14.7^{\mathrm{b}, \mathrm{c}}$ & $<0.001$ \\
\hline Carbohydrate ( $\%$ of energy) & $78.4^{\mathrm{a}, \mathrm{b}}$ & $74.0^{\mathrm{a}, \mathrm{c}}$ & $69.9^{\mathrm{b}, \mathrm{c}}$ & $<0.001$ \\
\hline
\end{tabular}

Data are $\mathrm{n}(\%)$ or mean \pm standard deviation.

${ }^{a} P<0.05 v s$. T1 and T2 of daily oral calcium intake by one-way ANOVA with Tukey multiple comparison post hoc analysis.

${ }^{\mathrm{b}} P<0.05 v s$. T1 and T3 of daily oral calcium intake by one-way ANOVA with Tukey multiple comparison post hoc analysis.

${ }^{\mathrm{c}} P<0.05$ vs. T2 and T3 of daily oral calcium intake by one-way ANOVA with Tukey multiple comparison post hoc analysis.

To convert $25(\mathrm{OH}) \mathrm{D}$ to nanomoles per liter, multiply by 2.496 . To convert parathyroid hormone (PTH) to nanograms per liter, multiply by 1. F, Female; HDL, high-density lipoprotein; M, male; ALP, alkaline phosphatase; AST, aspartate aminotransferase; ALT, alanine aminotransferase.

A Regular exercise is indicated as 'yes' when a subject performed vigorous exercise on a regular basis (for more than 20 min at a time and more than three times per week).

B Clerical work included sales and service, clerical staff, administration, and specialists. Manual work included agriculture, forestry, fishery, manual labor, engineering, assembling, and technical work.

${ }^{\mathrm{C}}$ Daily energy and nutrient intakes were assessed using the 24-h recall method of the Nutrition Survey of KNHANES IV. Dietary information was available for 1339 participants (sarcopenia, $\mathrm{n}=59$; non-sarcopenia, $\mathrm{n}=1280$ ) 
Table 2 Metabolic and dietary characteristics of participants with and without sarcopenia

\begin{tabular}{|c|c|c|c|}
\hline Variable & $\begin{array}{l}\text { With sarcopenia } \\
\qquad(\mathrm{n}=59)\end{array}$ & $\begin{array}{l}\text { Without sarcopenia } \\
\qquad(\mathrm{n}=1280)\end{array}$ & $p$-value \\
\hline Daily oral calcium intake $(\mathrm{mg} / \mathrm{dL})$ & $316.37 \pm 252.39$ & $414.65 \pm 316.10$ & 0.005 \\
\hline Age (yr) & $72.68 \pm 6.18$ & $69.96 \pm 6.72$ & 0.002 \\
\hline $\operatorname{Sex}(\mathrm{M} / \mathrm{F})$ & $33 / 26$ & $559 / 681$ & 0.169 \\
\hline BMI $\left(\mathrm{kg} / \mathrm{m}^{2}\right)$ & $23.2 \pm 1.4$ & $22.0 \pm 2.0$ & $<0.001$ \\
\hline Waist circumference $(\mathrm{cm})$ & $85.7 \pm 5.9$ & $79.3 \pm 7.3$ & $<0.001$ \\
\hline Total body fat mass $(\mathrm{kg})$ & $18.6 \pm 2.8$ & $14.0 \pm 4.1$ & $<0.001$ \\
\hline Total body fat percentage $(\%)$ & $33.7 \pm 5.9$ & $25.9 \pm 7.1$ & $<0.001$ \\
\hline $\operatorname{ASM}(k g)$ & $14.6 \pm 3.2$ & $16.9 \pm 3.9$ & $<0.001$ \\
\hline ASM/Wt (\%) & $25.6 \pm 3.1$ & $30.5 \pm 4.1$ & $<0.001$ \\
\hline Total cholesterol (mg/dL) & $192.7 \pm 40.0$ & $189.9 \pm 36.6$ & 0.598 \\
\hline HDL-cholesterol (mg/dL) & $48.4 \pm 12.7$ & $50.3 \pm 12.1$ & 0.258 \\
\hline Triglycerides (mg/dL) & $149.0 \pm 63.0$ & $138.6 \pm 93.4$ & 0.412 \\
\hline Fasting glucose (mg/dL) & $110.7 \pm 40.3$ & $102.8 \pm 29.8$ & 0.158 \\
\hline HOMA-IR & $4.9 \pm 15.2$ & $2.2 \pm 1.5$ & 0.209 \\
\hline $25(\mathrm{OH}) \mathrm{D}(\mathrm{ng} / \mathrm{mL})$ & $17.1 \pm 6.8$ & $20.5 \pm 7.4$ & 0.001 \\
\hline PTH (ng/liter) & $74.5 \pm 70.7$ & $69.2 \pm 28.4$ & 0.580 \\
\hline Regular exercise $^{\mathrm{a}}(\%)$ & $25.4 \%$ & $21.1 \%$ & 0.587 \\
\hline Vitamin supplement use (\%) & $21.1 \%$ & $25.4 \%$ & 0.424 \\
\hline \multicolumn{4}{|l|}{ Dietary intake $^{\mathrm{b}}$} \\
\hline Total energy (kcal/d) & $1434 \pm 538.0$ & $1650 \pm 679.8$ & 0.016 \\
\hline Protein (\% of energy) & 13 & 12 & 0.347 \\
\hline Fat ( $\%$ of energy) & 12 & 12 & 0.756 \\
\hline Carbohydrate ( $\%$ of energy) & 74 & 74 & 0.861 \\
\hline
\end{tabular}

To convert 25(OH)D to nanomoles per liter, multiply by 2.496. To convert parathyroid hormone (PTH) to nanograms per liter, multiply by 1 . F, Female; HDL, high-density lipoprotein; M, male; ALP, alkaline phosphatase; AST, aspartate aminotransferase; ALT, alanine aminotransferase; ASM, Appendicular skeletal muscle mass; HOMA-IR, homeostasis model assessment estimate of insulin resistance.

a Regular exercise is indicated as 'yes' when a subject performed vigorous exercise on a regular basis (for more than 20 min at a time and more than three times per week).

${ }^{b}$ Daily energy and nutrient intakes were assessed using the 24-h recall method of the Nutrition Survey of KNHANES IV. Dietary information was available for 1339 participants (sarcopenia, $\mathrm{n}=59$; non-sarcopenia, $\mathrm{n}=1280$ )

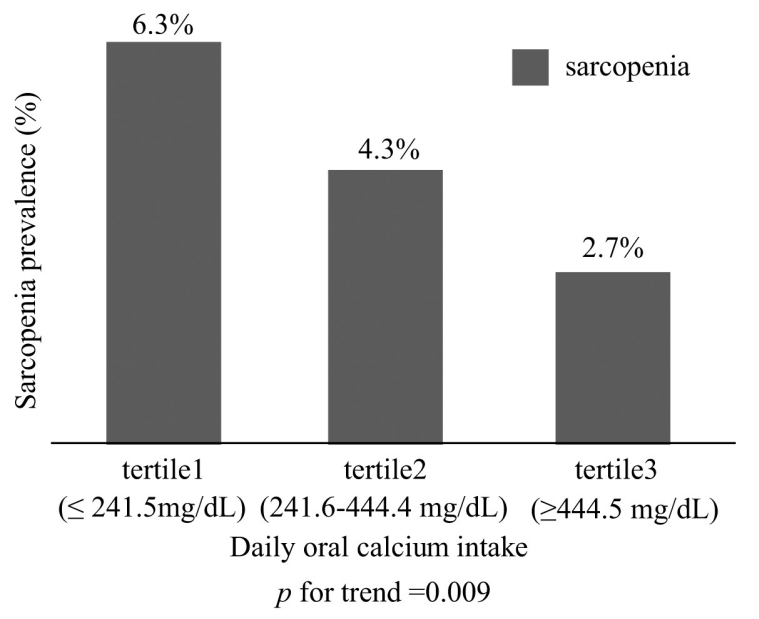

Fig. 1 Unadjusted prevalence of sarcopenia by daily oral calcium intake tertile

\section{Discussion}

We found a strong inverse association between daily calcium intake and sarcopenia in non-obese, older Korean adults. This association was independent of age, sex, BMI, total energy intake, serum 25(OH)D status, lifestyle factors (alcohol consumption, smoking, and regular exercise), and occupation. This association was particularly strong in subjects in the highest tertile for daily calcium intake when compared to subjects in the lowest tertile for daily calcium intake. Therefore, our data suggest that a relatively lower dietary calcium intake ( $278 \mathrm{mg}$ per day) is associated with an increased risk of sarcopenia in non-obese, older Korean adults.

This study has important clinical implications. 
Table 3 Adjusted ORs and 95\% CIs for sarcopenia according to daily oral calcium intake

\begin{tabular}{lcccc}
\hline & \multicolumn{3}{c}{ Daily oral calcium intake tertile } & \\
\cline { 2 - 4 } & $\mathrm{T} 1(\leq 241.5 \mathrm{mg} / \mathrm{dL})$ & $\mathrm{T} 2(241.6-444.4 \mathrm{mg} / \mathrm{dL})$ & $\mathrm{T} 3(\geq 444.5 \mathrm{mg} / \mathrm{dL})$ & \\
& $\mathrm{N}=447$ & $\mathrm{~N}=445$ & $0.413(0.207-0.823)$ & 0.010 \\
\hline Unadjusted & $1(\mathrm{ref})$ & $0.667(0.367-1.214)$ & $0.430(0.210-0.880)$ & 0.019 \\
Model 1 & $1(\mathrm{ref})$ & $0.696(0.377-1.285)$ & $0.380(0.183-0.790)$ & 0.008 \\
Model 2 & 1 (ref) & $0.590(0.316-1.104)$ & $0.391(0.163-0.940)$ & 0.033 \\
Model 3 & 1 (ref) & $0.613(0.296-1.270)$ & $0.259(0.087-0.768)$ & 0.014 \\
Model 4 & 1 (ref) & $0.620(0.280-1.374)$ & & \\
\hline
\end{tabular}

Model 1 adjusted for age and sex

Model 2 adjusted for age, sex, and BMI

Model 3 adjusted for age, sex, BMI, total energy intake, serum 25(OH)D, HOMA-IR, regular exercise, occupation, region, smoking, alcohol drinking, and vitamin/ mineral supplement use.

Model 4 adjusted for age, sex, BMI, total energy intake, serum 25(OH)D, HOMA-IR, regular exercise, occupation, region, smoking, alcohol drinking, and vitamin/ mineral supplement use, parathyroid hormone, and total fat mass.

Many reports have indicated that changes in body weight, and in particular fat mass, are associated with daily calcium intake [4-6]. Older adults have less muscle and bone mass, expanded extracellular fluid volumes, and reduced body cell mass compared to younger adults [19]. Non-fat components of body composition play critical roles in the health of elderly people [19]. In particular, skeletal muscle atrophy, or "sarcopenia," is highly prevalent in the elderly population and is strongly associated with disability, independent of morbidity. To our knowledge, this is the first crosssectional study to evaluate the relationship between skeletal muscle mass, separate from fat mass, and daily calcium intake.

This is the only study to report an association between daily calcium intake and sarcopenia, after correcting for total energy intake and serum $25(\mathrm{OH}) \mathrm{D}$ status. We can only hypothesize about the mechanisms by which calcium intake might alter the risk of developing sarcopenia in older Korean adults. First, lower dietary calcium intakes can lead to increased concentrations of $1,25(\mathrm{OH})_{2} \mathrm{D}$ and parathyroid hormone, which in turn may increase intracellular calcium concentrations [4, 27]. Alteration in calcium signaling may play a role in regulating muscle contractile force in differentiated muscle fibers. More recently, altered calcium homeostasis was found to be associated with skeletal muscle weakness during the aging process [20].

Second, low calcium intakes may increase the rate of lipogenesis and inhibit lipolysis, consequently leading to increased adiposity. Increased fat infiltration and fatty degeneration in skeletal muscle may cause subsequent atrophy of skeletal muscle, resulting in impaired muscular performance in older individuals. In our study, calcium intake was negatively correlated with total body fat mass and positively correlated with appendicular skeletal mass.

Previous studies have shown the significant role of serum 25(OH)D status and calcium intake in fat and muscle mass [21-23]. Recently, Kim et al. reported an inverse association between serum 25(OH)D levels and sarcopenia. Their data also support the putative role of serum $25(\mathrm{OH}) \mathrm{D}$ in preventing sarcopenia, which may contribute to a process by which serum $25(\mathrm{OH}) \mathrm{D}$ deficiency leads to physical disability [22]. In addition, high calcium diets have been found to regulate body weight and the accumulation of fat in adipocytes [7, 8]. Two possible mechanisms for an effect of dietary calcium on body weight have been suggested. First, calcium may combine with fatty acids in the intestine to form insoluble soaps that are therefore not absorbed [24]. Increased dietary calcium may decrease body fat stores by decreasing the absorption of fat. Second, some researchers have proposed that low dietary calcium intakes lead to increased adipocyte triglyceride deposition $[25,26]$.

The major strength of this study is that we used data from a large representative sample of older adults in the general population in Korea. Moreover, we evaluated body composition by dual-energy X-ray absorptiometry (DXA). DXA provides a higher degree of precision with a single measurement and can exactly locate where fat or muscle is distributed throughout the body. Because it produces reliable results, DXA is emerging as the new gold standard for body composition assessment $[25,26]$. These data provide the 
first assessment of the associations between sarcopenia and low calcium intake, body composition, and aging in an older population using DXA. Our study sample was confined to non-obese subjects. Numerous studies have demonstrated significant inverse associations between daily calcium intake and body weight and body fat, which is why we only included participants with a BMI between 18 and $25 \mathrm{~kg} / \mathrm{m}^{2}$. We demonstrated that daily calcium intake is associated with sarcopenia independent of body weight in a non-obese, elderly Korean sample.

There are several limitations to our study. First, the cross-sectional nature of this study makes it impossible to identify any cause-effect relationships. Second, data on vitamins or calcium supplements, prescribed medications, and comorbidities were not available. Third, we used a 24-hour recall method to assess dietary variables, but a single 24-hour recall may not be representative of habitual diet. Fourth, sarcopenia is an evolving concept and the current definition of sarcopenia includes both loss of muscle strength and of muscle mass. However, we relied on muscle mass only to define sarcopenia in the current study, hence the risk of overestimating the prevalence of sarcopenia. Fifth, all participants were Korean, and there is the possibility of selection bias. The mean daily dietary calcium intake in the present study was only $386 \mathrm{mg}$ /day: relatively low compared to other studies. Therefore, our study is limited in generalizing the results to the worldwide population.

In conclusion, the present study is the largest population-based study to examine the association between dietary calcium intake and sarcopenia. Daily calcium intake, corrected for total energy intake and serum 25 $(\mathrm{OH}) \mathrm{D}$ status, is significantly lower in subjects with sarcopenia than in those without. Our results suggest that there is a strong inverse association between daily calcium intake and sarcopenia in non-obese, older Korean adults.

\section{Disclosure Summary}

The authors have nothing to disclose.

\section{References}

1. Jacqmain M, Doucet E, Despres JP, Bouchard C, Tremblay A (2003) Calcium intake, body composition, and lipoprotein-lipid concentrations in adults. Am J Clin Nutr 77: 1448-1452.

2. Loos RJ, Rankinen T, Leon AS, Skinner JS, Wilmore JH, et al. (2004) Calcium intake is associated with adiposity in Black and White men and White women of the HERITAGE Family Study. J Nutr 134: 1772-1778.

3. Zemel MB (2004) Role of calcium and dairy products in energy partitioning and weight management. $\mathrm{Am} \mathrm{J}$ Clin Nutr 79: 907S-912S.

4. Parikh SJ, Yanovski JA (2003) Calcium intake and adiposity. Am J Clin Nutr 77: 281-287.

5. Barba G, Russo P (2006) Dairy foods, dietary calcium and obesity: a short review of the evidence. Nutr Metab Cardiovasc Dis 16: 445-451.

6. Rosenblum JL, Castro VM, Moore CE, Kaplan LM (2012) Calcium and vitamin D supplementation is associated with decreased abdominal visceral adipose tissue in overweight and obese adults. Am J Clin Nutr 95: 101108.

7. Shi H, Dirienzo D, Zemel MB (2001) Effects of dietary calcium on adipocyte lipid metabolism and body weight regulation in energy-restricted aP2-agouti transgenic mice. FASEB J 15: 291-293.

8. Teegarden D, Zemel MB (2003) Dairy product components and weight regulation: symposium overview. $J$
Nutr 133: 243S-244S.

9. Kyle UG, Genton L, Hans D, Karsegard VL, Michel JP, et al. (2001) Total body mass, fat mass, fat-free mass, and skeletal muscle in older people: cross-sectional differences in 60-year-old persons. J Am Geriatr Soc 49: 1633-1640.

10. Rossi A, Fantin F, Di Francesco V, Guariento S, Giuliano $\mathrm{K}$, et al. (2008) Body composition and pulmonary function in the elderly: a 7-year longitudinal study. Int $J$ Obes (Lond) 32: 1423-1430.

11. Abellan van Kan G (2009) Epidemiology and consequences of sarcopenia. J Nutr Health Aging 13: 708712.

12. Evans WJ (2010) Skeletal muscle loss: cachexia, sarcopenia, and inactivity. Am J Clin Nutr 91: 1123S$1127 \mathrm{~S}$.

13. Choi HS, Oh HJ, Choi H, Choi WH, Kim JG, et al. (2011) Vitamin D insufficiency in Korea--a greater threat to younger generation: the Korea National Health and Nutrition Examination Survey (KNHANES) 2008. $J$ Clin Endocrinol Metab 96: 643-651.

14. Matthews DR, Hosker JP, Rudenski AS, Naylor BA, Treacher DF, et al. (1985) Homeostasis model assessment: insulin resistance and beta-cell function from fasting plasma glucose and insulin concentrations in man. Diabetologia 28: 412-419.

15. Heymsfield SB, Smith R, Aulet M, Bensen B, Lichtman 
S, et al. (1990) Appendicular skeletal muscle mass: measurement by dual-photon absorptiometry. Am J Clin Nutr 52: 214-218.

16. Janssen I, Heymsfield SB, Ross R (2002) Low relative skeletal muscle mass (sarcopenia) in older persons is associated with functional impairment and physical disability. J Am Geriatr Soc 50: 889-896.

17. Lim S, Kim JH, Yoon JW, Kang SM, Choi SH, et al. (2010) Sarcopenic obesity: prevalence and association with metabolic syndrome in the Korean Longitudinal Study on Health and Aging (KLoSHA). Diabetes Care 33: 1652-1654.

18. Muscaritoli M, Anker SD, Argiles J, Aversa Z, Bauer JM, et al. (2010) Consensus definition of sarcopenia, cachexia and pre-cachexia: joint document elaborated by Special Interest Groups (SIG) "cachexia-anorexia in chronic wasting diseases" and "nutrition in geriatrics". Clin Nutr 29: 154-159.

19. Baumgartner RN (2000) Body composition in healthy aging. Ann N Y Acad Sci 904: 437-448.

20. Brotto M (2011) Aging, sarcopenia and store-operated calcium entry: a common link? Cell Cycle 10: 42014202.

21. Chung M, Balk EM, Brendel M, Ip S, Lau J, et al. (2009) Vitamin D and calcium: a systematic review of health outcomes. Evid Rep Technol Assess (Full Rep) Aug: 1-420.
22. Kim MK, Baek KH, Song KH, Il Kang M, Park CY, et al. (2011) Vitamin D deficiency is associated with sarcopenia in older Koreans, regardless of obesity: the Fourth Korea National Health and Nutrition Examination Surveys (KNHANES IV) 2009. J Clin Endocrinol Metab 96: 3250-3256.

23. Soares MJ, Chan She Ping-Delfos W, Ghanbari MH (2011) Calcium and vitamin D for obesity: a review of randomized controlled trials. Eur J Clin Nutr 65: 9941004.

24. Denke MA, Fox MM, Schulte MC (1993) Short-term dietary calcium fortification increases fecal saturated fat content and reduces serum lipids in men. J Nutr 123: 1047-1053.

25. Cummings NK, James AP, Soares MJ (2006) The acute effects of different sources of dietary calcium on postprandial energy metabolism. Br J Nutr 96: 138-144.

26. Melanson EL, Donahoo WT, Dong F, Ida T, Zemel MB (2005) Effect of low- and high-calcium dairy-based diets on macronutrient oxidation in humans. Obes Res 13: 2102-2112.

27. McCarty MF, Thomas CA (2003) PTH excess may promote weight gain by impeding catecholamine-induced lipolysis-implications for the impact of calcium, vitamin D, and alcohol on body weight. Med Hypotheses Nov-Dec;61(5-6): 535-542. 